\title{
Dynamic behavior of double-substrate interactive model
}

\author{
Sheng-Chi $\mathrm{Wu}^{\mathrm{a}, 1, *}$, Shun-Hsiung Shih ${ }^{\mathrm{b}, 2}$, Hwai-Shen Liu ${ }^{\mathrm{b}, 3}$ \\ ${ }^{a}$ Department of Biotechnology, Fooyin University, Ta-Liao Hsiang, Kaohsiung Hsien 831, Taiwan \\ ${ }^{\mathrm{b}}$ Department of Chemical Engineering, National Taiwan University, Taipei 106, Taiwan \\ Received 24 April 2006; accepted 3 August 2006
}

\begin{abstract}
Although modeling microbial growth with one limiting substrate such as Monod model is a common practice, microbes very often consume multiple substances from the environment for their growth. Therefore, the extension of current model to a multi-substrate analysis is needed for practical applications. The dynamic behavior of a double-substrate interactive growth model with Andrew's substrate inhibition model is theoretically discussed in this article. The yield factors considered are either a constant or a linear function of limiting substrate. The simulation indicated that there could be at least three non-trivial steady-state solutions with similar dynamic behavior for all the three cases, i.e. substrate without inhibition, one or both substrate with inhibition. The steady state of highest productivity is always a stable one. The steady state of the lowest productivity could change from a stable mode to an unstable mode while increasing dilution rate. And the limit cycle (sustained oscillation) could appear during the transition. The other steady state is always unstable. For the cases of one or both limiting substrates inhibition, the fourth steady state could appear, and it is always unstable.
\end{abstract}

(C) 2007 Taiwan Institute of Chemical Engineers. Published by Elsevier B.V. All rights reserved.

Keywords: Microbial growth; Modeling; Double-substrate; Dynamic behavior

\section{Introduction}

Cells grow by taking up multiple substances from the environment. These nutrients include different carbon sources and a wide variety of components such as nitrogen, amino acids, vitamins and so on. Therefore, the possibility of multisubstrate limitations is quite frequently encountered in practice. In fact, the situation of multiple limiting substrates is noted in some experiments (Court and Pirt, 1981; Machado and Grady, 1989; Panikov, 1979).

Continuous culture is an important tool to produce the desired products under optimal operating conditions and also to determine the response of microorganisms to the environment. Double-substrate limiting growth is the simplest condition of multiple limiting substrates and needs to be examined first. Those cases have been discussed for the microorganisms of interest, including variation of cellular composition (Boer et al., 2003), accumulation of storage compound (Durner et al.,

\footnotetext{
* Corresponding author. Tel.: +8867 781151x643; fax: +88677862707

E-mail address: dr009@mail.fy.edu.tw (S.-C.Wu).

1 吳聲祺

2 施順雄

${ }^{3}$ 劉懷勝
}

2001), changing of cell metabolism (Weusthuis et al., 1994) and excretion of metabolic intermediates (Jung et al., 2001). These detail results were reviewed by Zinn et al. (2004). From the viewpoint of maximum production, accurate control of the growth nutrients in the culture broth is very important and needs suitable kinetic model, especially in the case of multiple limiting substrates. Therefore, the characteristics of the proposed model need to be analyzed more clearly.

Some mathematical models are developed to describe the growth rate of a biological population with two or more limiting substrates. Bader (1978) classified the mathematical models of double-substrate limiting growth making into two categories. One is the non-interactive model which the growth rate of the biological population is controlled by one limiting substrate at one time. The other, interactive model, describes the growth rate as a function of both two limiting substrates at the same time. Chen and Christensen (1985) developed a unified model which is based on the probability. Cybernetic hypothesis, developed by Ramkrishna and coworkers (Dhurjati et al., 1985; Kompala et al., 1984, 1986), described the response of microorganisms tend to maximize yield among possible substrates, especially, in continuous culture. The situation that nutrients were not necessarily substitutable was noted by Baltzis and Fredrickson (1988) both experimentally and theoretically, which later 


\section{Nomenclature}

$a, b \quad$ constant in the formulation of yield factor $Y_{2}$

$A, B \quad$ functions defined in Eq. (7)

$C_{\mathrm{s} 1}, C_{\mathrm{s} 2}$ two limiting substrate concentrations

$C_{\mathrm{x}} \quad$ cell concentration

$C_{\text {xo }} \quad$ scaling factor

$D$ dimensionless dilution rate

$D_{\mathrm{o}} \quad$ dilution rate

$K_{\mathrm{i} 1}, K_{\mathrm{i} 2}$ model constants defined in Eq. (1)

$K_{\text {s1 }}, K_{\text {s2 }}$ model constants defined in Eq. (1)

$K_{1}, K_{2}$ dimensionless model constant defined in Eq. (3)

$P_{\mathrm{m} 1}, P_{\mathrm{m} 2}, P_{z}$ functions defined in Eqs. (6a) and (6b)

$S_{1}, S_{2}$ dimensionless two limiting substrate concentrations

$t \quad$ dimensionless time

$t_{\mathrm{o}} \quad$ time

$X \quad$ dimensionless cell concentration

$y_{1}, y_{2}$ yield factors for two limiting substrates

$Y_{1}, Y_{2}$ dimensionless yield factors for two limiting substrates

Greek symbols

$\lambda \quad$ eigenvalue

$\mu \quad$ dimensionless specific cell growth rate

$\mu_{\mathrm{s}} \quad$ specific cell growth rate

$\mu_{\mathrm{sm}} \quad$ maximum specific cell growth rate

\section{Subscript}

$\mathrm{F} \quad$ the value in inlet stream

resulted in an extension of cybernetic model by Pavlou and Fredrickson (1989). Haas (1994) also proposed a formulation which was based on non-interactive growth process occurring among perfectly substitutable nutrients.

In this article, the dynamic behavior of a double-substrate limited interactive growth model with Andrew's substrate inhibition model (Andrews, 1968) for each substrate proposed by Liu et al. (1993) is discussed, i.e.

$\mu_{\mathrm{s}}=\frac{\mu_{\mathrm{sm}} C_{\mathrm{s} 1} C_{\mathrm{s} 2}}{\left(\left(K_{\mathrm{s} 1}+C_{\mathrm{s} 1}+C_{\mathrm{s} 1}^{2}\right) / K_{\mathrm{i} 1}\right)\left(\left(K_{\mathrm{s} 2}+C_{\mathrm{s} 2}+C_{\mathrm{s} 2}^{2}\right) / K_{\mathrm{i} 2}\right)}$

where $\mu_{\mathrm{s}}$ is the specific growth rate, $\mu_{\mathrm{sm}}$ the maximum specific growth rate, $C_{\mathrm{s} 1}$ and $C_{\mathrm{s} 2}$ the two limiting substrate concentrations, $K_{\mathrm{s} 1}$ and $K_{\mathrm{s} 2}$ the model constants, and $K_{\mathrm{i} 1}$ and $K_{\mathrm{i} 2}$ are substrate inhibition constants. When $K_{\mathrm{i} 1}$ and $K_{\mathrm{i} 2}$ approach to infinity, inhibition effect is neglected and this model is reduced to MeGee et al.'s model (1972) which is simply a product of two Monod models for each substrate. Microbial growth, such as Lacfobacillus plantarum (Lee et al., 1976), Saccharomyces cereoisiue (Borzani et al., 1977), Saccharomyces carlsbergensis (Finn and Wilson, 1954) and Klebsiella aerogenes (Harrison and Pirt, 1967), in continuous cultures occasionally exhibits oscillatory phenomena. The simple model failed to explain the observed oscillatory behavior in the chemostat. The model with constant yield term had been evidenced that it could not have any periodic solutions (Crooke et al., 1980). In 1982, the studies of Crooke and Tanner (1982) and Agrawal et al. (1982) first reported that if the yield coefficient increases linearly with substrate concentration, the stable steady state may undergo the Hopf bifurcation and a limit cycle may appear. The single specie fermentation process had been investigated and continue until now (Zhu and Huang, 2006). In this study, we discussed a double-substrate limiting growth model and the yield factor either a constant or a linear function of limiting substrate was used.

\section{Theoretical formulation}

\subsection{Mathematical model}

For a continuous bioreactor, system variables are cell concentration $\left(C_{\mathrm{x}}\right)$ and two limiting substrate concentrations $\left(C_{\mathrm{s} 1}, C_{\mathrm{s} 2}\right)$. The governing equations with control variables, dilution rate $\left(D_{\mathrm{o}}\right)$ and two feed substrate concentrations $\left(C_{\mathrm{s} 1 \mathrm{~F}}\right.$, $C_{\mathrm{s} 2 \mathrm{~F}}$ ), can be described in the dimensionless forms as:

$\frac{\mathrm{d} X}{\mathrm{~d} t}=\mu X-D X$

$\frac{\mathrm{d} S_{1}}{\mathrm{~d} t}=-\frac{\mu X}{Y_{1}}+D\left(S_{1 \mathrm{~F}}-S_{1}\right)$

$\frac{\mathrm{d} S_{2}}{\mathrm{~d} t}=-\frac{\mu X}{Y_{2}}+D\left(S_{2 \mathrm{~F}}-S_{2}\right)$

where $X=\left(C_{\mathrm{x}} / C_{\mathrm{xo}}\right), S_{1}=\left(C_{\mathrm{s} 1} / K_{\mathrm{s} 1}\right), S_{2}=\left(C_{\mathrm{s} 2} / K_{\mathrm{s} 2}\right), D=\left(D_{\mathrm{o}} / \mu_{\mathrm{sm}}\right)$, $\mu=\left(\mu_{\mathrm{s}} / \mu_{\mathrm{sm}}\right), t=\left(t_{\mathrm{o}} / \mu_{\mathrm{sm}}\right), \quad Y_{1}=\left(K_{\mathrm{s} 1} y_{1} / C_{\mathrm{xo}}\right), \quad Y_{2}=\left(K_{\mathrm{s} 2} y_{2} / C_{\mathrm{xo}}\right)$, $S_{1 \mathrm{~F}}=\left(C_{\mathrm{s} 1 \mathrm{~F}} / K_{\mathrm{s} 1}\right), S_{2 \mathrm{~F}}=\left(C_{\mathrm{s} 2 \mathrm{~F}} / K_{\mathrm{s} 2}\right)$.

The parameters $Y_{1}$ and $Y_{2}$ are the dimensionless yield factors (cell/substrate) for the two limiting substrates, respectively. $C_{\mathrm{xo}}$ is a scaling factor and $t_{\mathrm{o}}$ is time. The corresponding dimensionless specific growth rate in Eq. (1) becomes:

$\mu=\frac{S_{1} S_{2}}{\left(1+S_{1}+K_{1} S_{1}^{2}\right)\left(1+S_{2}+K_{2} S_{2}^{2}\right)}$

where $K_{1}=\left(K_{\mathrm{s} 1} / K_{\mathrm{i} 1}\right)$ and $K_{2}=\left(K_{\mathrm{s} 2} / K_{\mathrm{i} 2}\right)$.

The yield factor considered in this study was either a constant $\left(Y_{1}\right)$ or a linear function of limiting substrate $\left(Y_{2}=a+b S_{2}\right)$ (Essajee and Tanner, 1979).

\subsection{Steady state}

Besides the washout steady state $\left(X, S_{1}, S_{2}\right)=\left(0, S_{1 \mathrm{~F}}, S_{2 \mathrm{~F}}\right)$, the non-trivial solution can be expressed as:

$\mu=D$

$X=Y_{1}\left(S_{1 \mathrm{~F}}-S_{1}\right)$

$X=Y_{2}\left(S_{2 \mathrm{~F}}-S_{2}\right)$

By substituting the growth Eq. (3) into Eq. (4a), one may have:

$\frac{1}{D}=\left(\frac{1}{S_{1}}+1+K_{1} S_{1}\right)\left(\frac{1}{S_{2}}+1+K_{2} S_{2}\right)$ 
And the following equation can be obtained from Eqs. (4b) and (4c) by eliminating $X$.

$S_{1}=S_{1 \mathrm{~F}}-\frac{Y_{2}\left(S_{2 \mathrm{~F}}-S_{2}\right)}{Y_{1}}=S_{1 \mathrm{~F}}-\frac{\left(a+b S_{2}\right)\left(S_{2 \mathrm{~F}}-S_{2}\right)}{Y_{1}}$

Eq. (5a) is a hyperbola in the $S_{1}-S_{2}$ phase plane when $K_{1}=K_{2}=0$ as discussed by Liu et al. (1993) and Eq. (5b) is a parabolic curve with a right open end and the vertex is at $P_{\mathrm{m}}$ $\left(P_{\mathrm{m} 1}, P_{\mathrm{m} 2}\right)$, where

$P_{\mathrm{m} 1}=-\frac{\left(a+b S_{2 \mathrm{~F}}\right)^{2}-4 b S_{1 \mathrm{~F}} Y_{1}}{4 b Y_{1}} \quad$ and $\quad P_{\mathrm{m} 2}=-\frac{a-b S_{2 \mathrm{~F}}}{2 b}$

The curve described by Eq. (5b) intersects with the line $S_{2}=0$ at $\left(P_{z}, 0\right)$, where

$P_{z}=S_{1 \mathrm{~F}}-\frac{a S_{2 \mathrm{~F}}}{Y_{1}}$

in the $S_{1}-S_{2}$ phase plane, the steady-state solutions are the intersection points of these two curves, i.e. Eqs. (5a) and $(5 b)$.

\subsection{Local stability and bifurcation theory}

The criterion of a local stability is that the real parts for all eigenvalues of the Jacobian matrix at the respective steady state are negative (Verhulst, 1990). The Jacobian matrix can be expressed as:

$\mathbf{J}=\left(\begin{array}{ccc}\mu-D & -\frac{\partial \mu}{\partial S_{1}} X & -\frac{\partial \mu}{\partial S_{2}} X \\ -\frac{\mu}{Y_{1}} & -\frac{\partial \mu X}{\partial S_{1} Y_{1}}-D & -\frac{\partial \mu}{\partial S_{2}} \frac{X}{Y_{1}} \\ -\frac{\mu}{Y_{1}} & -\frac{\partial \mu}{\partial S_{1}} \frac{X}{Y_{2}} & -\frac{\partial\left(\mu / Y_{2}\right)}{\partial S_{2}} X-D\end{array}\right)$

The local stability of the continuous bioreactor with double limiting substrates can be analysis as follows:

(I) For non-trivial solution:

The eigenvalues are the roots of the characteristic equation of Eqs. (2a)-(2c), i.e. $(\mathbf{J}-\lambda \mathbf{I})=0$.

$(\lambda+D)\left(\lambda^{2}+A \lambda+B\right)=0$

in which

$$
\begin{aligned}
A= & D+\left(\left(\frac{\partial \mu}{\partial S_{2}}\right)\left(\frac{1}{Y_{2}}\right)+\left(\frac{\partial \mu}{\partial S_{1}}\right)\left(\frac{1}{Y_{1}}\right)\right) X \\
& -\left(\frac{\mu X}{Y_{2}^{2}}\right)\left(\frac{\partial Y_{2}}{\partial S_{2}}\right) \\
B= & D X\left(\left(\frac{\partial \mu}{\partial S_{2}}\right)\left(\frac{1}{Y_{2}}\right)+\left(\frac{\partial \mu}{\partial S_{1}}\right)\left(\frac{1}{Y_{1}}\right)\right) \\
& -\frac{\mu X^{2}}{Y_{1} Y_{2}^{2}}\left(\frac{\partial \mu}{\partial S_{1}}\right)\left(\frac{\partial Y_{2}}{\partial S_{2}}\right)
\end{aligned}
$$

When $Y_{2}$ is a constant, the three roots of the characteristic equation are:

$-D,-D,-\left(\left(\frac{\partial \mu}{\partial S_{2}}\right)\left(\frac{1}{Y_{2}}\right)+\left(\frac{\partial \mu}{\partial S_{1}}\right)\left(\frac{1}{Y_{1}}\right)\right) X$

In this case, there is no Hopf bifurcation possible (Leah, 1988).

When $Y_{2}$ is a linear function of limiting substrate, the local stability of non-washout steady state can be determined by the sign of

$$
\left(\frac{\partial \mu}{\partial S_{2}}\right)\left(\frac{1}{Y_{2}}\right)+\left(\frac{\partial \mu}{\partial S_{1}}\right)\left(\frac{1}{Y_{1}}\right)
$$

Since

$\frac{\partial \mu}{\partial S_{1}}=\frac{1-K_{1} S_{1}^{2}}{\left(1+S_{1}+K_{1} S_{1}^{2}\right)^{2}} \frac{S_{2}}{\left(1+S_{2}+K_{2} S_{2}^{2}\right)}$

and

$\frac{\partial \mu}{\partial S_{2}}=\frac{1-K_{2} S_{2}^{2}}{\left(1+S_{2}+K_{2} S_{2}^{2}\right)^{2}} \frac{S_{1}}{\left(1+S_{1}+K_{1} S_{1}^{2}\right)}$

the group

$\left(\frac{\partial \mu}{\partial S_{2}}\right)\left(\frac{1}{Y_{2}}\right)+\left(\frac{\partial \mu}{\partial S_{1}}\right)\left(\frac{1}{Y_{1}}\right)$

is always positive if $1>K_{1} S_{1}^{2}$ and $1>K_{2} S_{2}^{2}$. Please note that the case of $K_{1}=K_{2}=0$ (no substrate inhibition) always satisfies $1>K_{1} S_{1}^{2}$ and $1>K_{2} S_{2}^{2}$. In this case, the steady state is stable because of the negative eigenvalue. With substrate inhibition, the steady state may be either stable or unstable. However, when $Y_{2}$ is a linear function of the second limiting substrate, the three roots of the characteristic equation are:

$-D, \frac{-A+\sqrt{A^{2}-4 B}}{2}, \frac{-A-\sqrt{A^{2}-4 B}}{2}$

Therefore, limit cycle is possible if $A=0$ and $B>0$ according to Hopf bifurcation theory.

(II) For trivial solution (washout):

The three eigenvalues are:

$\lambda=-D,-D, \mu-D$

It is a stable steady state when the dilution rate is greater than the specific growth rate $(D>\mu)$, and an unstable node otherwise.

\section{Result and discussion}

Three cases, (I) both substrates without inhibition effect, (II) substrate inhibition for one substrate only, and (III) substrate inhibition for both limiting substrates, are discussed in the following results using numerical simulations according to the substrates with or without inhibition effect. The $S_{1}-S_{2}$ phase plane is used to exam the possible classes of steady states and 


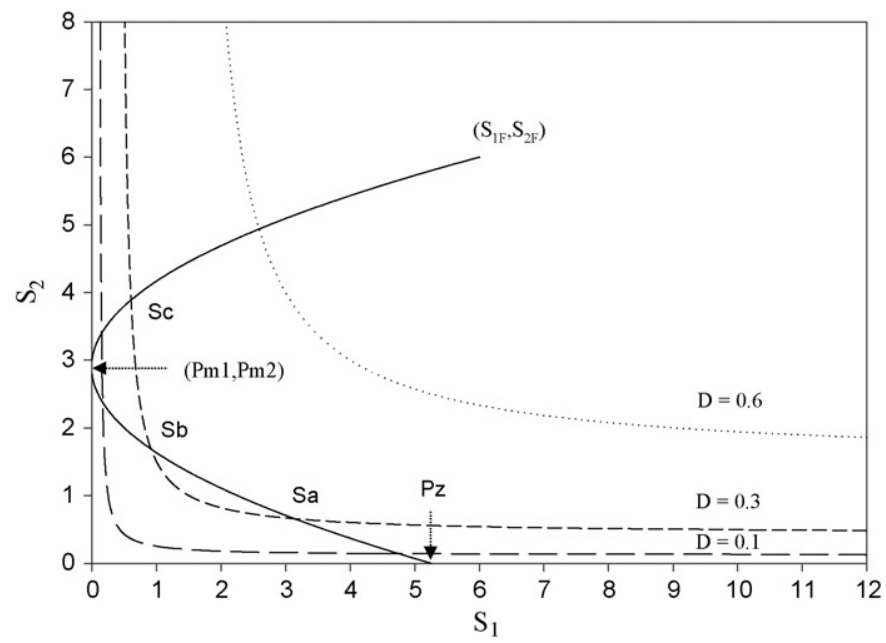

Fig. 1. Steady-state solutions from Eq. (5a) (dash line with different dilution rate) and Eq. (5b) (solid line) for both substrates without inhibition $\left(Y_{1}=8\right.$, $a=1, b=5, S_{1 \mathrm{~F}}=6, S_{2 \mathrm{~F}}=6$ and $\left.K_{1}=K_{2}=0\right)$.

bifurcation diagram commonly used is performed to classify the possible phase plots with dilution rate.

\subsection{Case (I): both substrates without inhibition effect}

Fig. 1 shows the steady-state solutions in $S_{1}-S_{2}$ phase plane if both of the limiting substrates in the interactive model
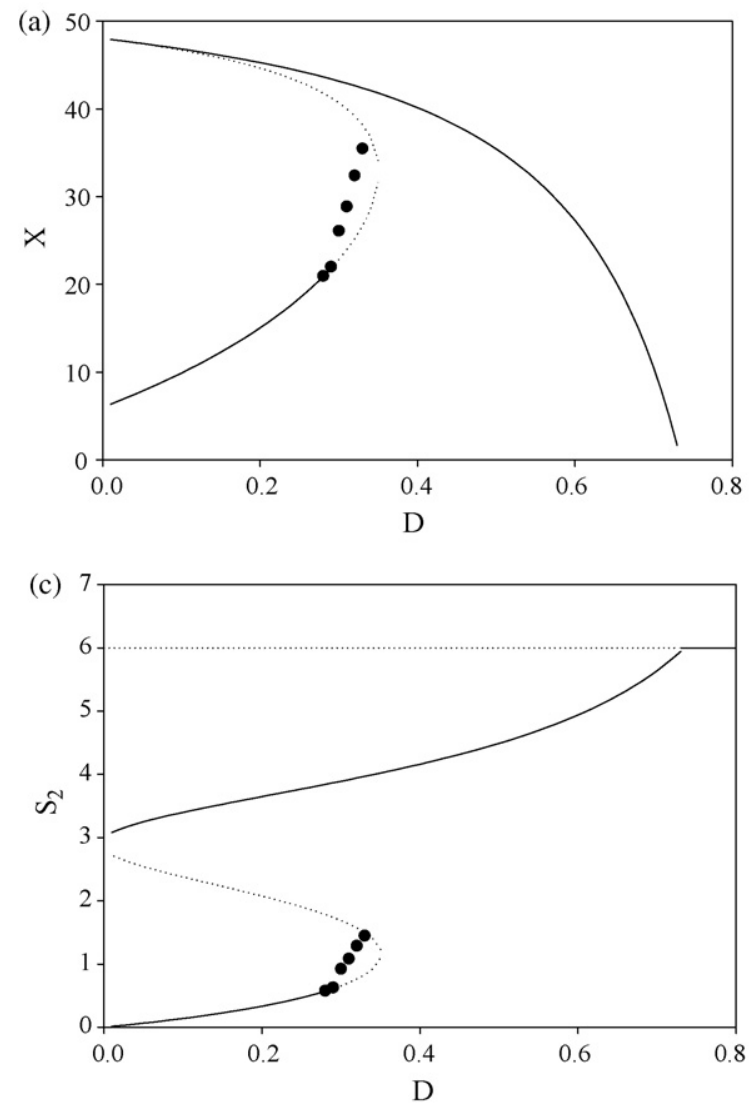

follow the Monod models. The solid line is the feeding parabolic curve as presented in Eq. (5b) which can be moved by one of the operating factors, feeding concentration. The lines are growth hyperbola curves from Eq. (5a) with $K_{1}=K_{2}=0$ which is decided by the other operating factor, dilution rate. For feeding curve, the characteristic of the parabolic curve with a right open end and the vertex $P_{\mathrm{m}}\left(P_{\mathrm{m} 1}, P_{\mathrm{m} 2}\right)$ is important to the appearance of the possible classes of steady states. The growth hyperbola curves (Fig. 1) with three dilution rates, $D=0.1,0.3$ and 0.6, move upwards as the dilution rate increases. If the hyperbola curves are above the feed point $\left(S_{1 \mathrm{~F}}, S_{2 \mathrm{~F}}\right)$, a hyperbola curves and parabolic curve never intersect with each other. Therefore, only the trivial steady state is exist, that is the washout condition. Critical dilution rate can be obtained where the growth curve passes the feeding concentration $\left(S_{1 \mathrm{~F}}, S_{2 \mathrm{~F}}\right)$. Except for trivial solution, there are three possible classes of steady states which are dependent upon the feeding concentration, i.e. the feeding parabolic curves. There is at most one nonwashout steady state for whole range of dilution rates when $P_{\mathrm{m} 2}$ (the coordinate of vertex of parabolic curve) is negative. This steady state is the highest one located on the upper half portion of the parabolic curve and always exists except the washout steady state. When $P_{\mathrm{m} 2}$ is positive (i.e. $S_{2 \mathrm{~F}}>a / b$ by Eq. (6a)), there could be either one or three steady states, determined by using the Eq. (5b) with $S_{2}=0$. This intersection of $S_{1}$ is noted as $P_{z}$ (Fig. 1). When $P_{z}$ is positive $\left(\left(Y_{1} / a\right) S_{1 \mathrm{~F}}>S_{2 \mathrm{~F}}\right)$, i.e. the lower half portion of feeding parabolic curves across the first
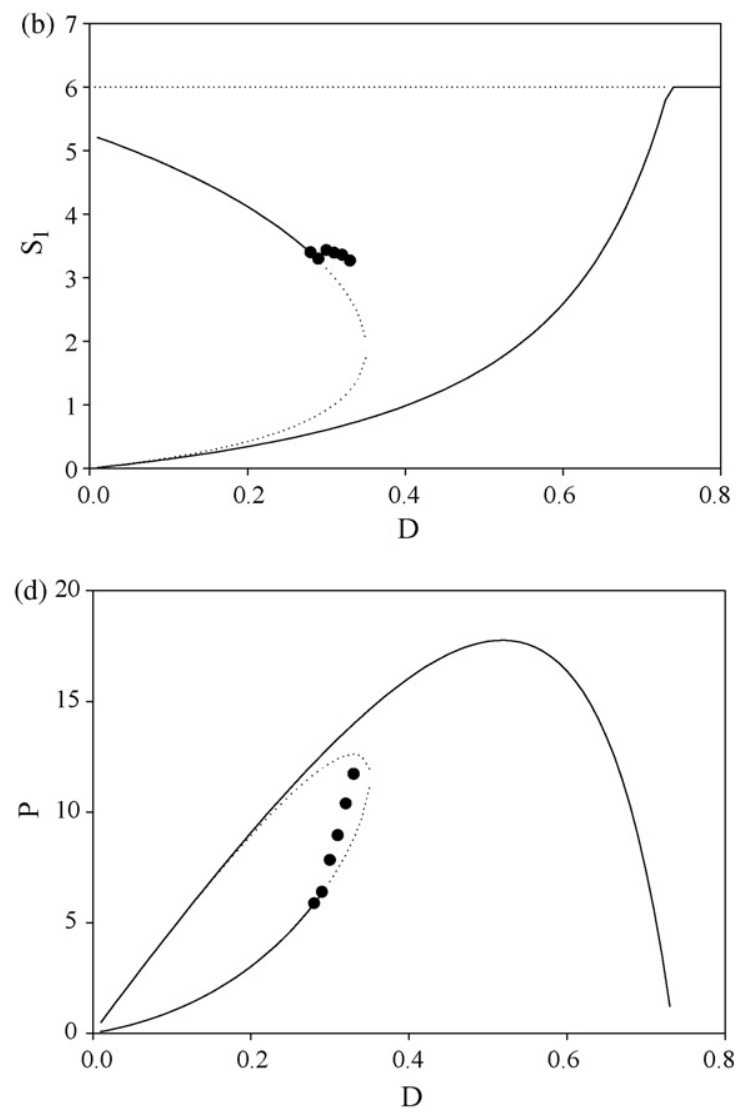

Fig. 2. (a) Bifurcation diagram of both substrates without inhibition for cell, (b) substrate 1, (c) substrate 2 , and (d) productivity $\left(Y_{1}=8, a=1, b=5, S_{1 \mathrm{~F}}=6, S_{2 \mathrm{~F}}=6\right.$ and $K_{1}=K_{2}=0$ ). 
quadrant, there are three steady-state solutions. Otherwise $\left(P_{z}<0\right)$, only one steady state can be obtained. In other words, if $P_{\mathrm{m} 1}$ is positive (i.e. $S_{1 \mathrm{~F}}>\left(\left(a+b S_{2 \mathrm{~F}}\right)^{2} / 4 b Y_{1}\right)$ by Eq. (6a)), there are three steady-state solutions in the middle range of the dilution rate since $P_{z}$ is always positive. It should be noticed that the lowest steady state only exist in the case of three steadystate solutions and in the lower range of the dilution rate.

For the purpose of illustration, the parameters $\left(Y_{1}=8, a=1\right.$, $b=5, S_{1 \mathrm{~F}}=6$ and $S_{2 \mathrm{~F}}=6$ ) are chosen so that three non-trivial steady states exist and the vertex is in the second quadrant. The bifurcation diagram for cell and two limiting substrates in Fig. 1 with three steady states are shown in Fig. 2(a)-(c). The number of steady state when dilution rate approaches zero is three because of positive $P_{\mathrm{m} 2}$ and $P_{z}$. In Fig. 2(c), the variation of three non-washout steady states $\left(S_{\mathrm{a}}, S_{\mathrm{b}}\right.$ and $\left.S_{\mathrm{c}}\right)$ with dilution rate is shown with three regions of $S_{2}, 0-1.1\left(S_{\mathrm{a}}\right), 1.1-2.8\left(S_{\mathrm{b}}\right)$ and 3$6\left(S_{\mathrm{c}}\right)$. The highest steady state for $S_{2}\left(S_{\mathrm{c}}\right)$ is always a stable attractor. The washout steady state is a saddle when $S_{\mathrm{c}}$ exists at the same time and becomes stable when $S_{\mathrm{c}}$ disappeared $(D>\mu)$. This transition point is the critical operating dilution rate. Above the transition point, only one stable is exist, i.e. the washout steady state. $S_{\mathrm{b}}$ is always unstable (a saddle point) and Hopf bifurcation phenomenon appears in the lower steady state $\left(S_{\mathrm{a}}\right) . S_{\mathrm{a}}$ is a stable spiral steady state in lower dilution rate and is changed to unstable spiral when dilution rate increases to exceed bifurcation value. The limit cycle which is a circle in the three dimensional space appears in the transition. This stable limit cycle has an unstable spiral inside. When dilution rate increases, the average radius of the cycle becomes bigger. Up to maximum extend the cycle will break. Only one attracting steady state $\left(S_{\mathrm{c}}\right)$ in the state space is presented in higher dilution rate region and the maximum productivity which is the cell concentration multiplying by dilution rate is also in this region (Fig. 2(d)). The classification of possible phase plots is shown in Table 1 where eight different situations are classified.

\subsection{Case (II): substrate inhibition for one substrate only}

For the inhibition effect of substrate 1 only, the steady-state solution(s) formulated in Eqs. (5a) and (5b) with $K_{2}=0$ are shown in Fig. 3. The solid line is also the feeding parabolic curves as discussed in case (I). The others are growth curves

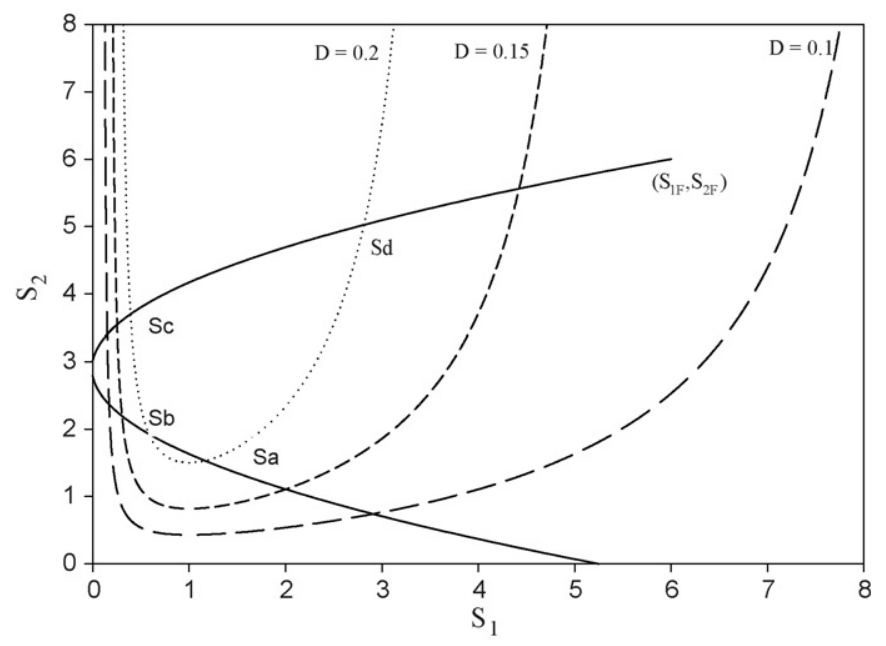

Fig. 3. Steady-state solutions from Eq. (5a) (dash line with different dilution rate) and Eq. (5b) (solid line) for only one substrate inhibition $\left(Y_{1}=8, a=1\right.$, $b=5, S_{1 \mathrm{~F}}=6, S_{2 \mathrm{~F}}=6, K_{1}=1$ and $K_{2}=0$ ).

forming the U-shape curves. When the dilution rate increase, the U-shape moves upward resulting in the width of the shape decreases (Liu et al., 1993). The minimum of the U-shape curves in which the slope equals to zero can be obtained from the solutions of the first derivative of Eq. (5a) and $S_{1}=\sqrt{K_{1}}$. Using the function substitute into Eq. (5a), one value of the concentration of substrate 2 can be obtained since $K_{2}=0$. Therefore, both sides of the minimum of the U-shape curves are monotonically increased to infinity which is limited by two vertical asymptotes. The steady-state solutions are the intersection points of the two curves, feeding and dilution rate curves. The number of non-washout steady state in this case is similar with case (I) except $S_{\mathrm{d}}$ shown in Fig. 3. Since substrate inhibition causes right hand side of U-shape dilution rate curves, the feeding parabolic curve intersects with Ushape, generating an additional and maximum steady state. Therefore, the maximum number of non-washout steady state is two when the vertex in the third or fourth quadrants $\left(P_{\mathrm{m} 2}<0\right)$. When the vertex is in the first or second quadrant $\left(P_{\mathrm{m} 2}>0\right)$, non-washout steady state is four $\left(S_{\mathrm{a}}, S_{\mathrm{b}}, S_{\mathrm{c}}\right.$ and $\left.S_{\mathrm{d}}\right)$ in the condition of $P_{z}>0$ and two while $P_{z}$ is below zero.

Table 1

The classification of possible phase plots with dilution rate as parameter for both substrates without inhibition effect

\begin{tabular}{|c|c|c|c|c|c|c|c|c|}
\hline & \multicolumn{8}{|l|}{ Case } \\
\hline & A & $\mathrm{B}$ & $\mathrm{C}$ & $\mathrm{D}$ & $\mathrm{E}$ & $\mathrm{F}$ & $\mathrm{G}$ & $\mathrm{H}$ \\
\hline Region of dilution rate & $0-0.144$ & $0.144-0.292$ & $0.292-0.33$ & $0.33-0.35$ & $0.35-0.52$ & $0.52-0.572$ & $0.572-0.735$ & $0.735-0.8$ \\
\hline Stable washout & 0 & 0 & 0 & 0 & 0 & 0 & 0 & 1 \\
\hline Unstable washout & 1 & 1 & 1 & 1 & 1 & 1 & 1 & 0 \\
\hline Stable node & 2 & 1 & 1 & 1 & 1 & 0 & 1 & 0 \\
\hline Saddle & 1 & 1 & 1 & 1 & 0 & 0 & 0 & 0 \\
\hline Stable spiral & 0 & 1 & 0 & 0 & 0 & 1 & 0 & 0 \\
\hline Unstable spiral & 0 & 0 & 1 & 1 & 0 & 0 & 0 & 0 \\
\hline Limit cycle & 0 & 0 & 1 & 0 & 0 & 0 & 0 & 0 \\
\hline Total & 4 & 4 & 5 & 4 & 2 & 2 & 2 & 1 \\
\hline
\end{tabular}

$Y_{1}=8, a=1, b=5, S_{1 \mathrm{~F}}=6, S_{2 \mathrm{~F}}=6$ and $K_{1}=K_{2}=0$. 
(a)

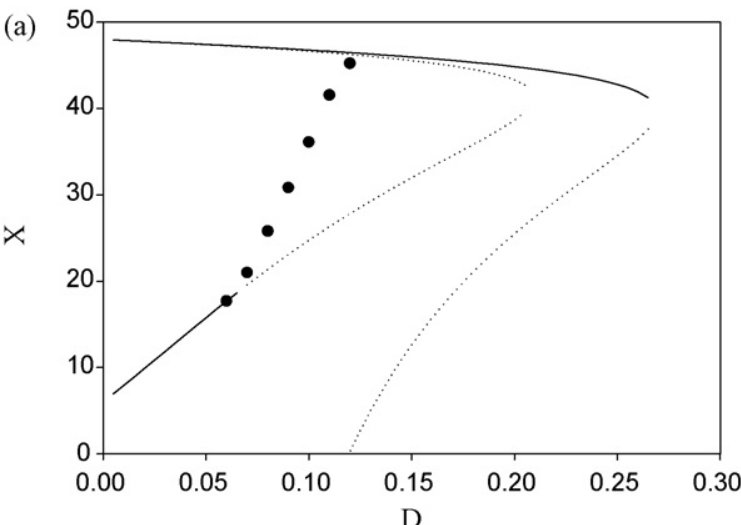

(c)

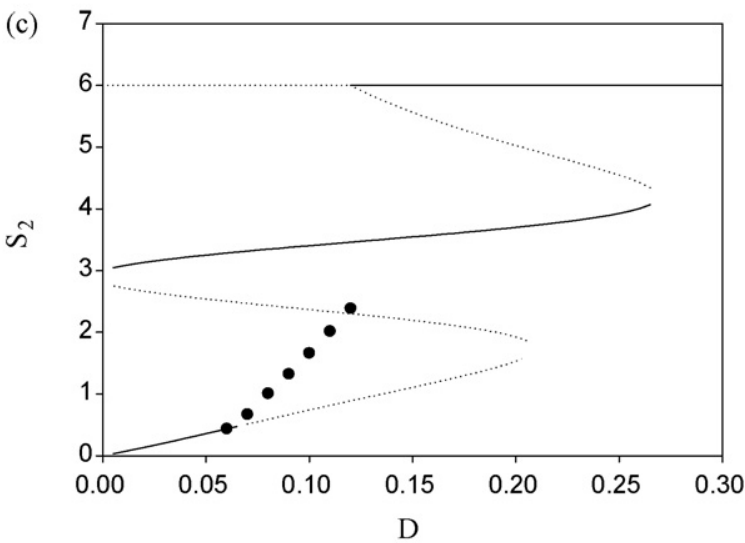

(b)

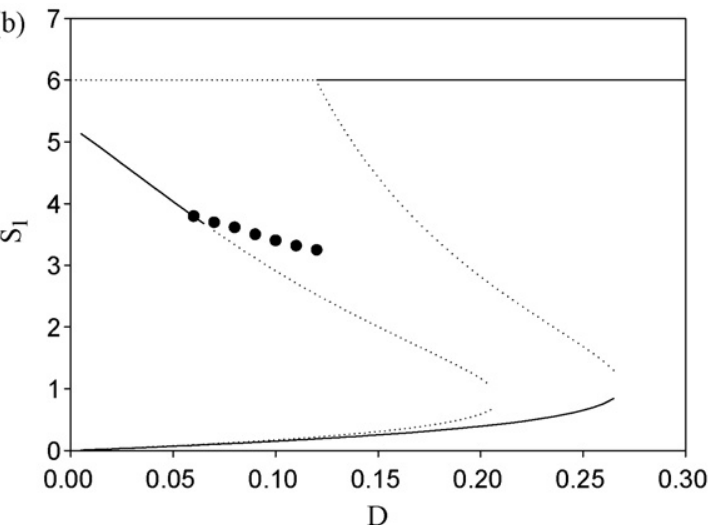

(d)

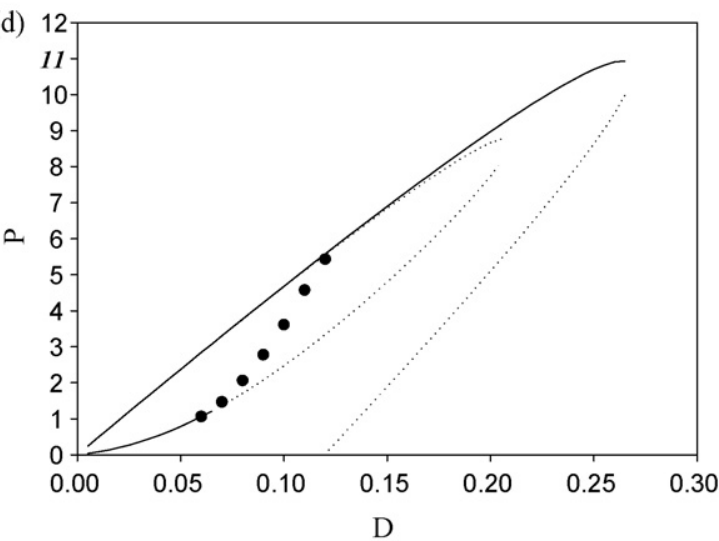

Fig. 4. (a) Bifurcation diagram of only one substrate inhibition for cell, (b) substrate 1, (c) substrate 2, and (d) productivity $\left(Y_{1}=8, a=1, b=5, S_{1 \mathrm{~F}}=6, S_{2 \mathrm{~F}}=6\right.$, $K_{1}=1$ and $\left.K_{2}=0\right)$.

Comparing with case (I), the parameters are the same except additional parameter $K_{1}=1$.

The bifurcation diagram as a function of dilution rate is shown in Fig. 4(a)-(c) for cell and two limiting substrates, respectively. Since $P_{\mathrm{m} 2}$ and $P_{z}$ are positive, there are three nonwashout steady states when dilution rate approaches to zero due to the selected parameters. The fourth steady state appears in the middle range of the dilution rate and disappears at the critical dilution rate $(D=\mu)$. The variations of $S_{2}$ for the four non-washout steady states are indicated in Fig. 3(c) with four regions of $S_{2}, 0-1.7\left(S_{\mathrm{a}}\right), 1.7-2.8\left(S_{\mathrm{b}}\right), 3.0-4.2\left(S_{\mathrm{c}}\right)$ and 4.2-6.0 $\left(S_{\mathrm{d}}\right)$. The behaviors of three steady state $S_{\mathrm{a}}, S_{\mathrm{b}}$ and $S_{\mathrm{c}}$ are similar to case (I); $S_{\mathrm{c}}$ is a stable attractor, $S_{\mathrm{b}}$ is a saddle and $S_{\mathrm{a}}$ is changed from stable to unstable with increasing dilution rate. The stable limit cycle also appears in $S_{\mathrm{a}}$ and starts at the bifurcation value then breaks before $S_{\mathrm{d}}$ appears. $S_{\mathrm{d}}$ does not exist when dilution rate is lower since its steady-state value of $\left(S_{1}, S_{2}\right)$ is greater than $\left(S_{\mathrm{F} 1}\right.$, $S_{\mathrm{F} 2}$ ). It is always an unstable steady state. The washout steady state is unstable in the lower range of dilution rate, and becomes unstable as dilution rate increases resulting in the formation of the $S_{\mathrm{d}} . S_{\mathrm{d}}$ is disappeared at the critical dilution rate $(D=\mu)$. Productivity, defined by the product of cell concentration and dilution rate, is shown in Fig. 4(d) as a function of dilution rate. The maximum productivity is operated in the dilution rate $D_{\mathrm{m}}$

Table 2

The classification of possible phase plots with dilution rate as parameter for only one substrate inhibition

\begin{tabular}{|c|c|c|c|c|c|c|c|}
\hline & \multicolumn{7}{|l|}{ Case } \\
\hline & A & B & $\mathrm{C}$ & $\mathrm{D}$ & E & $\mathrm{F}$ & G \\
\hline Region of dilution rate & $0-0.028$ & $0.028-0.068$ & $0.068-0.1$ & $0.1-0.12$ & $0.12-0.209$ & $0.209-0.269$ & $0.269-0.3$ \\
\hline Stable washout & 0 & 0 & 0 & 0 & 1 & 1 & 1 \\
\hline Unstable washout & 1 & 1 & 1 & 1 & 0 & 0 & 0 \\
\hline Stable node & 2 & 1 & 1 & 1 & 1 & 1 & 0 \\
\hline Saddle & 1 & 1 & 1 & 1 & 2 & 1 & 0 \\
\hline Stable spiral & 0 & 1 & 0 & 0 & 0 & 0 & 0 \\
\hline Unstable spiral & 0 & 0 & 1 & 1 & 1 & 0 & 0 \\
\hline Limit cycle & 0 & 0 & 1 & 0 & 0 & 0 & 0 \\
\hline Total & 4 & 4 & 5 & 4 & 5 & 3 & 1 \\
\hline
\end{tabular}

$Y_{1}=8, a=1, b=5, S_{1 \mathrm{~F}}=6, S_{2 \mathrm{~F}}=6, K_{1}=1$ and $K_{2}=0$. 


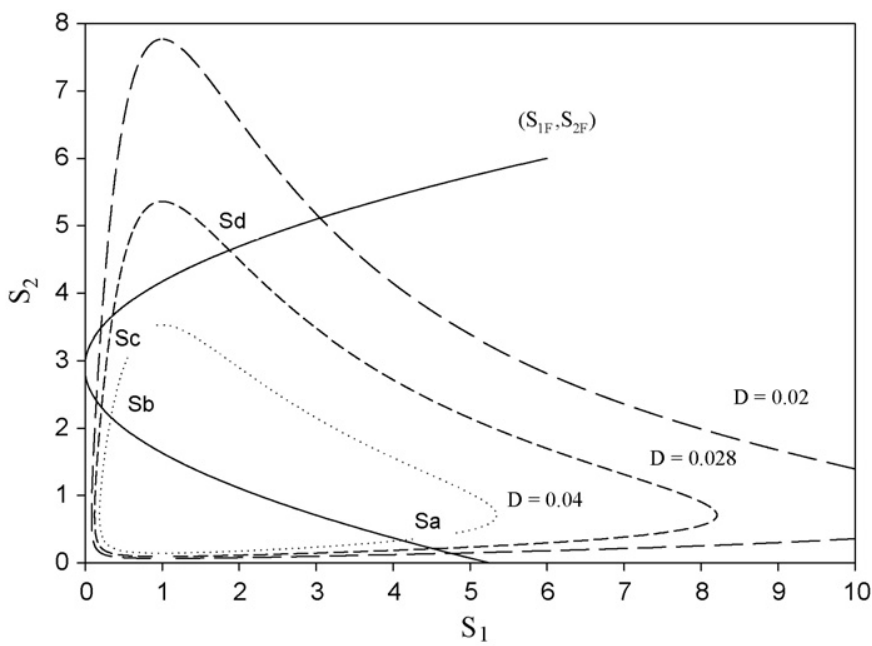

Fig. 5. Steady-state solutions from Eq. (5a) (dash line with different dilution rate) and Eq. (5b) (solid line) for both substrates with inhibition $\left(Y_{1}=8, a=1\right.$, $b=5, S_{1 \mathrm{~F}}=6, S_{2 \mathrm{~F}}=6, K_{1}=1$ and $K_{2}=2$ ).

and the steady-state value of $S_{2}$ will be $S_{\mathrm{c}}$ (Fig. 3). Slightly increasing dilution rate at $D_{\mathrm{m}}$ will lead to the steady state at only one stable attractor-washout. The washout steady state is the condition of loss of all cells at steady state that means the productivity is zero. Therefore, an accurate flow-rate controller is needed to avoid the disturbance of dilution rate which is function of flow rate in operating bioreactor. The seven different classes of phase plots are presented in Table 2 .
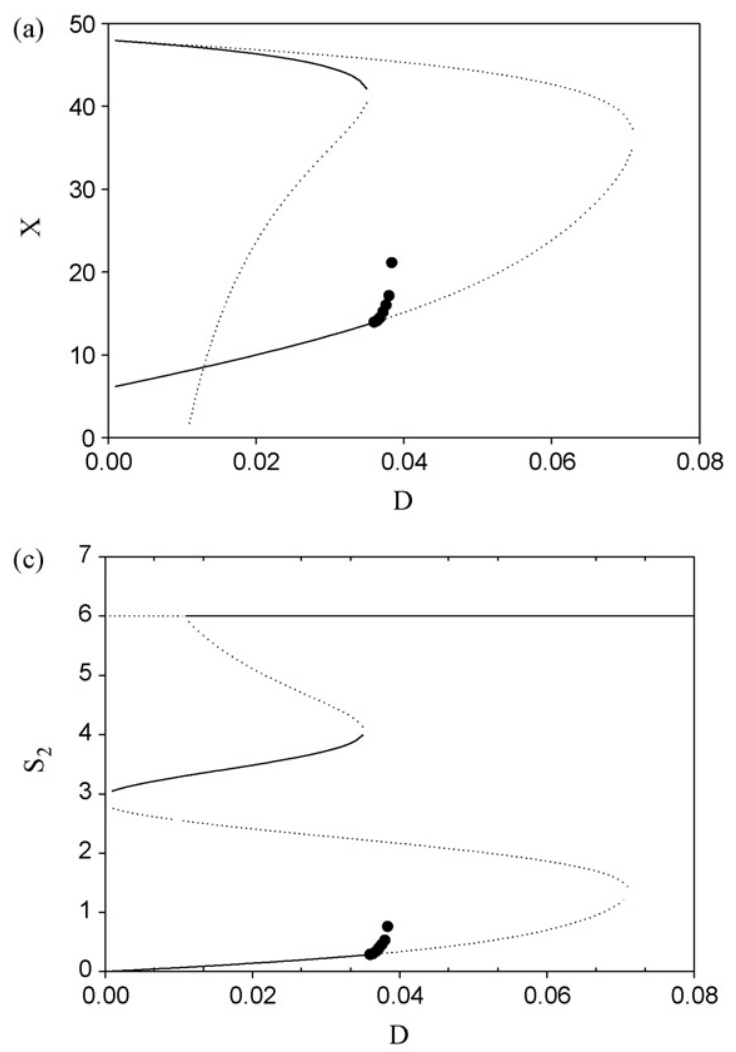

\subsection{Case (III): substrate inhibition for both limiting substrates}

When both of the limiting substrates in the interactive model produce an inhibition effect, the steady-state solution(s) is formulated in Eqs. (5a) and (5b) and shown in Fig. 5. Although the feeding parabolic curve (the solid line) is also the same as discussed in case (I), the growth curves become more complicated. The extreme of the curves which are the solutions of first derivative of Eq. (5a) can be obtained in $S_{1}=\sqrt{K_{1}}$ as in case (II), but when using the function substitute into Eq. (5a), the concentration of substrate 2 can be obtained two values since $K_{2} \neq 0$. Because $S_{1}$ and $S_{2}$ are symmetry in Eq. (5a), two extreme of the curves can be also obtained in $S_{2}=\sqrt{K_{2}}$. Therefore, the closed-loop contour curve is expressed in Fig. 5 for the growth curves. When the dilution rate increases, the closed-loop contour curve reduces its size and finally approaches a point, where the dilution rate is the limiting dilution rate. The number of steadystate solutions which are the intersection points of feeding and dilution rate curves is similar as case (II). Therefore, the additional steady state is obtained when compared with case (I) and the maximum number of non-washout steady state is two when the vertex is located in the third and fourth quadrants. When the vertex is located in the first or second quadrant, there are four steady states $S_{\mathrm{a}}, S_{\mathrm{b}}, S_{\mathrm{c}}$ and $S_{\mathrm{d}}$ in the condition of $P_{z}>0$ and two steady states while $P_{z}<0$.

The parameters in the bifurcation diagram shown in Fig. 6(a)-(c) are the same as case (II); however, the $K_{2}$ is 2
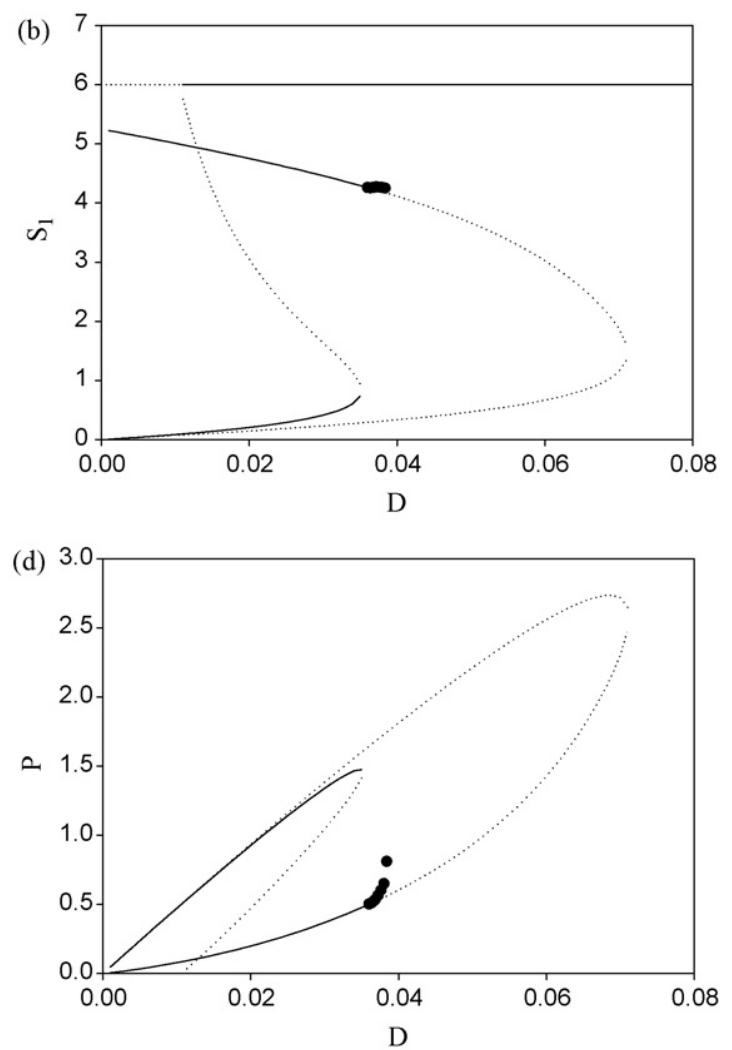

Fig. 6. (a) Bifurcation diagram of both substrates with inhibition for cell, (b) substrate 1, (c) substrate 2, and (d) productivity $\left(Y_{1}=8, a=1, b=5, S_{1 \mathrm{~F}}=6, S_{2 \mathrm{~F}}=6\right.$, $K_{1}=1$ and $K_{2}=2$ ). 
Table 3

The classification of possible phase plots with dilution rate as parameter for both limiting substrates with inhibition

\begin{tabular}{|c|c|c|c|c|c|c|c|c|}
\hline & \multicolumn{8}{|l|}{ Case } \\
\hline & A & B & $\mathrm{C}$ & $\mathrm{D}$ & $\mathrm{E}$ & $\mathrm{F}$ & G & $\mathrm{H}$ \\
\hline Region of dilution rate & $0-0.011$ & $0.011-0.024$ & $0.024-0.0352$ & $0.0352-0.0368$ & $0.0368-0.038$ & $0.038-0.052$ & $0.052-0.071$ & $0.071-0.08$ \\
\hline Stable washout & 0 & 1 & 1 & 1 & 1 & 1 & 1 & 1 \\
\hline Unstable washout & 1 & 0 & 0 & 0 & 0 & 0 & 0 & 0 \\
\hline Stable node & 2 & 2 & 1 & 0 & 0 & 0 & 0 & 0 \\
\hline Unstable node & 0 & 0 & 0 & 0 & 0 & 0 & 1 & 0 \\
\hline Saddle & 1 & 2 & 2 & 1 & 1 & 1 & 1 & 0 \\
\hline Stable spiral & 0 & 0 & 1 & 1 & 0 & 0 & 0 & 0 \\
\hline Unstable spiral & 0 & 0 & 0 & 0 & 1 & 1 & 0 & 0 \\
\hline Limit cycle & 0 & 0 & 0 & 0 & 1 & 0 & 0 & 0 \\
\hline Total & 4 & 5 & 5 & 3 & 4 & 3 & 3 & 1 \\
\hline
\end{tabular}

$Y_{1}=8, a=1, b=5, S_{1 \mathrm{~F}}=6, S_{2 \mathrm{~F}}=6, K_{1}=1$ and $K_{2}=2$.

instead. The stability of four steady states is similar to case (II) but the limit cycle is produced after $S_{\mathrm{d}}$ is disappeared. For productivity versus dilution rate (Fig. 6(d)), the difference of dilution rate between the maximum productivity and the limit cycle is very closure. It also needs to accurately control the dilution rate in order to obtain a maximum production due to hysteresis effect. The eight regions have been classified for different phase plots and are corrected in Table 3.

\section{Conclusion}

A typical plot to explain the effect of double substrates with one variable yield factor is given by using specific or specified values. The position of vertex (Eq. (6a)) and the intersection of $S_{1}$ (Eq. (6b)) in $S_{1}-S_{2}$ contour determine the maximum number of steady state and there are function of control variables $S_{1 \mathrm{~F}}$ and $S_{2 \mathrm{~F}}$. In the case of both substrates without inhibition effect (case (I)), at most one non-washout steady state is obtained for whole range of dilution rate when $P_{\mathrm{m} 2}$ (the coordinate of vertex of parabolic curve) is negative. When $P_{\mathrm{m} 2}$ is positive, there are three steady-state solutions if $P_{z}$ is positive and only one steady state if $P_{z}$ is negative. In the case of one substrate with inhibition (case (II)), the number of non-washout steady state is similar to case (I) except there is an additional steady state. Therefore, the maximum number of non-washout steady state is two when $P_{\mathrm{m} 2}<0$. If $P_{\mathrm{m} 2}>0$, there are four steady states in the condition of $P_{z}>0$ and two steady states while $P_{z}<0$. In the case (III) with substrate inhibition for both limiting substrates, the maximum number of non-washout steady state is the same as case (II) although the growth curve becomes a closed loop.

After simulation with many parameters, the steady state $S_{\mathrm{c}}$ is always a stable one and the maximum productivity is also operated in this condition. $S_{\mathrm{b}}$ and $S_{\mathrm{d}}$ (in cases (II) and (III)) are unstable and $S_{\mathrm{a}}$ which is the lowest steady state could be change from a stable mode to an unstable mode while dilution rate increases. The limit cycle (sustained oscillation) could appear during the transition. Because of the presence of hysteresis effect in the cases of substrate inhibition with one or two substrate, accurate control in operating bioreactor is needed to avoid the disturbance of dilution rate for producing maximum productivity.

\section{References}

Agrawal, P., C. Lee, H. C. Lim, and D. Ramkrishna, "Theoretical Investigations of Dynamic Behavior of Isothermal Continuous Stirred Tank Biological Reactors," Chem. Eng. Sci., 37, 453 (1982).

Andrews, J. F., "A Mathematical Model for the Continuous Culture of Microorganisms Utilizing Inhibitory Substrate," Biotechnol. Bioeng., 10, 707 (1968).

Bader, F. G., "Analysis of Double-Substrate Limited Growth," Biotechnol. Bioeng., 20, 183 (1978).

Baltzis, B. C. and A. G. Fredrickson, "Limitation of Growth Rate by Two Complementary Nutrients: Some Elementary but Neglected Considerations," Biotechnol. Bioeng., 31, 75 (1988).

Boer, V. M., J. H. de Winde, J. T. Pronk, and M. D. W. Piper, "The GenomeWide Transcriptional Responses of Saccharomyces cerevisiae Grown on Glucose in Aerobic Chemostat Cultures Limited for Carbon, Nitrogen, Phosphorus, or Sulfur," J. Biol. Chem., 278, 3265 (2003).

Borzani, W., R. E. Gregori, and M. L. R. Vairo, "Some Observations on Oscillatory Changes in the Growth Rate of Saccharomyces cerevisiae in Aerobic Continuous Undisturbed Culture,” Biotechnol. Bioeng., 19, 1363 (1977).

Chen, C. Y. and E. R. Christensen, "A Unified Theory for Microbial Growth under Multiple Nutrient Limitation," Water Res., 19, 791 (1985).

Court, J. R. and S. J. Pirt, “Carbon- and Nitrogen-Limited Growth of Pencillium chrysogenum in Fed Batch Culture: The Optimal Ammonium Ion Concentration for Penicillin Production," J. Chem. Technol. Biotechnol., 31, 235 (1981).

Crooke, P. S., C. J. Wei, and R. D. Tanner, "Effect of the Specific Growth Rate and Yield Expressions on the Existence of Oscillatory Behavior of a Continuous Fermentation Model," Chem. Eng. Commun., 6, 333 (1980).

Crooke, P. S. and R. D. Tanner, "Hopf Bifurcations for a Variable Yield Continuous Fermentation Model," Int. J. Eng. Sci., 20, 439 (1982).

Dhurjati, P. S., D. Ramkrishna, M. C. Flickinger, and G. T. Tsao, "A Cybernetic View of Microbial Growth: Modeling of Cells as Optimal Strategists," Biotechnol. Bioeng., 27, 1 (1985).

Durner, R., M. Zinn, B. Witholt, and T. Egli, “Accumulation of Poly[(R)-3Hydroxyalkanoates] in Pseudomonas oleovorans during Growth in Batch and Chemostat Culture with Different Carbon Sources," Biotechnol. Bioeng., 72, 278 (2001).

Essajee, C. K. and R. D. Tanner, "The Effect of Extracellular Variables on the Stability of the Continuous Baker's Yeast-Ethanol Fermentation Process," Process Biochem., 14, 16 (1979).

Finn, R. K. and R. E. Wilson, "Fermentation Process Control, Population Dynamics of a Continuous Propagator for Microorganisms," J. Agric. Food Chem., 2, 66 (1954).

Haas, C. N., "Unified Kinetic Treatment for Growth on Dual Nutrients," Biotechnol. Bioeng., 44, 154 (1994). 
Harrison, D. E. F. and S. J. Pirt, "The Influence of Dissolved Oxygen Concentration on the Respiration and Glucose Metabolism of Klebsiella aerogenes during Growth," J. Gen. Microbiol., 46, 193 (1967).

Jung, K., W. Hazenberg, M. Prieto, and B. Witholt, "Two-Stage Continuous Process Development for the Production of Medium-Chain-Length Poly(3hydroxyalkanoates)," Biotechnol. Bioeng., 72, 19 (2001).

Kompala, D. S., D. Ramkrishna, and G. T. Tsao, "Cybernetic Modeling of Microbial Growth on Multiple Substrates," Biotechnol. Bioeng., 26, 1272 (1984).

Kompala, D. S., D. Ramkrishna, N. B. Jansen, and G. T. Tsao, "Investigation of Bacterial Growth on Mixed Substrates: Experimental Evaluation of Cybernetic Models," Biotechnol. Bioeng., 28, 1044 (1986).

Leah, E. K., Mathematical Models in Biology, Random House, New York, U.S.A. (1988).

Lee, I. H., A. G. Fredrickson, and H. M. Tsuchiya, "Damped Oscillations in Continuous Culture of Lactobacillus plantarum," J. Gen. Microbiol., 93, 204 (1976).

Liu, H. S., S. S. Wang, and H. W. Hsu, "Double-Substrate Interactive Model," Chem. Eng. Sci., 48, 2169 (1993).

Machado, R. J. and C. P. L. Grady, Jr."Dual Substrate Removal by an Axenic Bacterial Culture," Biotechnol. Bioeng., 33, 327 (1989).
MeGee, R. D., III J. F. Drake, A. G. Fredrickson, and H. M. Tsuchiya, "Studies in Intermicrobial Symbiosis. Saccharomyces cerevisiae and Lactobacillus casei," Can. J. Microbiol., 18, 1733 (1972).

Panikov, N., "Steady State Growth Kinetics of Chlorella Vulgaris under Double Substrate (Urea and Phosphate) Limitation," J. Chem. Technol. Biotechnol., 29, 442 (1979).

Pavlou, S. and A. G. Fredrickson, "Growth of Microbial Populations in Nonminimal Media: Some Considerations for Modeling," Biotechnol. Bioeng., 34, 971 (1989).

Verhulst, F., Nonlinear Differential Equations and Dynamical Systems, Springer, Berlin, Germany (1990).

Weusthuis, R. A., W. Visser, J. T. Pronk, W. A. Scheffers, and J. P. van Dijken, "Effects of Oxygen Limitation on Sugar Metabolism in Yeasts: A Continuous-Culture Study of the Kluyver Effect," Microbiology, 140, 703 (1994).

Zhu, L. and X. Huang, "Multiple Limit Cycles in a Continuous Culture Vessel," Nonlinear Anal., 64, 887 (2006).

Zinn, M., B. Witholt, and T. Egli, "Dual Nutrient Limited Growth: Models, Experimental Observations, and Applications," J. Biotechnol., 113, 263 (2004).

\title{
雙限制基質交互作用下生物反應器之動態分析
}

\author{
吳聲祺 \\ 輔英科技大學生物技術系 \\ 施順雄 劉懷勝 \\ 台灣大學化學工程系
}

摘要

雖然目前常以單一限制基質來描述微生物成長, 但微生物總是從環境中攝取多種基質以利生長。因此, 將現存的模 式延伸到多基質有其實際的必要性。本文以 Andrew 的基質抑制模式, 來探討雙限制基質交互作用下生物反應器之動態行 為。模式中的兩個產率因子，一選擇為常數而另一為基質濃度之線性函數。對於所討論的三種情況而言，包括雙限制基 質均無抑制、單限制基質抑制及雙限制基質均有抑制現象, 數值分析顯示這三種情況均可得類似的至少三個非零解。其 中最高的穩態總是穩定且可產生最大產量, 而最低的穩態隨著稀釋速率增加從穩定態變化到非穩定態, 且在轉換過程中 出現極限圓。另一個穩態總是不穩定。對於一或二個基質抑制的情況, 產生了第四個穩態但總是不穩定。 